\title{
Avaliação multiprofissional de saúde em adolescentes de uma escola pública de Altamira-PA
}

\author{
Multiprofessional health assessment in adolescents of a \\ public school of Altamira-PA
}

\author{
Dinar Duarte Vasconcelos', Paulo Henrique Guerra ${ }^{2,3}$, Jeniffer Fagundes4, \\ Kharinni Uchôa Pereira ${ }^{5}$, Silvia Ribeiro Santos Araújo 6
'Programa de Pós-graduação em Ensino e Saúde na Amazônia, Universidade do Estado do Pará, Altamira (PA), Brasil.
${ }^{2}$ Universidade Federal da Fronteira Sul, Chapecó (SC), Brasil.
${ }^{3}$ Grupo de Estudos e Pesquisas Epidemiológicas em Atividade Física e Saúde, Universidade de São Paulo, São Paulo (SP), Brasil.
${ }^{4}$ Grupo Multidisciplinar de Pesquisa em Educação, Saúde e Meio Ambiente na Amazônia, Universidade do Estado do Pará, Altamira (PA), Brasil.
${ }^{5}$ Unidade de Ensino-Assistência de Fisioterapia e Terapia Ocupacional, Programa de Pós-graduação Ensino em Saúde na Amazônia, Universidade do Estado do Pará, Belém (PA), Brasil. \\ ${ }^{6}$ Faculdade de Educação Física, Universidade Federal de Mato Grosso, NAFIMES, Cuiabá (MT), Brasil.
}

Recebido: Jun., 25, 2018
Aceito: Fev., 12, 2019

\section{COMO CITAR ESTE ARTIGO} Vasconcelos DD, Guerra PH, Fagundes J, Pereira KU, Araújo SRS. Avaliação multiprofissional de saúde em adolescentes de uma escola pública de Altamira-PA. Interdisciplinary Journal of Health Education. 2020 Jan-Jun:5(1):46-52. https://doi.org/10.4322/ijhe.2020.006

\section{CORRESPONDÊNCIA}

Dinar Duarte Vasconcelos

Universidade do Estado do Pará,

Campus IX

Avenida Bom Jesus, s/n, Mutirão,

CEP 68377-000, Altamira (PA), Brasil divasconcelos@uepa.br

\section{FONTE DE FINANCIAMENTO}

Financiamento próprio.

\section{CONFLITO DE INTERESSE}

Os autores declaram não haver

conflito de interesse.

O estudo foi realizado em escola pública, Altamira (PA), Brasil.

Todos os autores leram e aprovam a versão final submetida ao Interdisciplinary Journal of Health Education (IJHE).

\section{RESUMO}

Contextualização: recomenda-se que a promoção da saúde no cenário escolar ocorra por meio de um processo de permanente monitoramento e avaliação das ações, partindo do diálogo entre os setores saúde e educação, favorecendo a logística necessária para sua execução, de forma lógica, factível no contexto do calendário letivo, sem desconsiderar a dinâmica escolar. Descrição da experiência: este texto relata uma atividade de caráter intersetorial, inserida no contexto do Programa Saúde na Escola (PSE), que envolveu ações conjuntas entre unidade escolar, coordenação de atenção básica (CAB) e a Universidade do Estado do Pará, em uma escola pública de ensino médio da cidade de Altamira-PA. O espaço escolar foi organizado em analogia ao modelo de atenção do Sistema Único de Saúde (SUS), de modo que os adolescentes passassem por um atendimento geral (Profissional de Educação Física, Enfermagem, agente comunitário de saúde, odontólogo) e, conforme a particularidade de determinados casos, encaminhados para atendimentos por especialidades (nutricionista, psicólogo e assistente social). A atividade permitiu realizar uma avaliação diagnóstica multiprofissional de saúde de adolescentes do ensino médio, e identificar fatores de riscos e/ou doenças que poderiam influenciar no desempenho escolar. Resultados e impactos: ampliação da possibilidade de acesso e do diagnóstico das condições de saúde dos adolescentes e encaminhamento via SUS para tratamento de agravos identificados. Considerações finais: percebendo-se a importância do desenvolvimento de ações em saúde voltadas às populações de adolescentes, espera-se que esta experiência possa servir de base para agregar ao debate. Em particular, cabe apontar que a CAB propiciou o acesso aos serviços, utilizando a ação como porta de entrada para o SUS, possibilitando a redução de riscos à saúde, aumento da produtividade, desempenho, resolutividade com garantia de acesso aos serviços.

PAlaVRAS-Chave: Saúde. Educação. Serviços de Saúde Escolar. Colaboração Intersetorial. Programa Saúde na Escola.

\section{ABSTRACT}

Contextualization: The recommendation is that health promotion in the school scenario occurs through a process in constant development, monitoring and evaluation of the actions, starting from the dialogue between the sectors of health and education, in order to think the logistics necessary for its execution, a rational and feasible way in the context of the school calendar, without disregarding the school dynamics. Description of the experience: this text reports an intersectoral activity, inserted in the context of the School Health Program (PSE), which involved joint actions among the school unit, basic care coordination (CAB) and the State University of Pará, in a public school of basic education of the city of Altamira-PA. The school space was organized in analogy to the Unified Health System (SUS) model of care, so that the adolescents underwent general care (Physical Education Professional, Nursing, community health agent, dentist) and according to the particularity (nutritionist, psychologist and social worker). The activity allowed to perform 
a multiprofessional diagnostic evaluation of high school adolescents' health and to identify risk factors and / or diseases that could affect school performance. Results and impacts: besides expanding the possibility of access and diagnosis of the adolescents' health conditions and referral services through SUS to treat identified diseases. Final considerations: Due to the importance of the development of health-oriented actions for adolescents, we expect that this experience could serve as a basis to add to the debate. In particular, it should be pointed out that the CAB provided access to services, using the action as a gateway to the SUS, enabling health risk reduction, productivity increase, performance, and resoluteness with guaranteed access to services.

KEYWORDS: Health. Education. School Health Service. Intersectoral Collaboration. Health at School Program.

\section{Contextualização}

O contexto escolar é um espaço de construção de saberes, desenvolvimento de atitudes, mudanças de hábitos, produção de sentidos e significados, privilegiado para ações de educação e saúde. É desejável que ações de promoção de saúde na escola sejam conduzidas com permanente monitoramento e avaliação, partindo do diálogo entre os setores saúde e educação, favorecendo a logística necessária para sua execução, de forma lógica e factível no contexto do calendário letivo, sem desconsiderar a dinâmica da escola ${ }^{1}$.

Nessa conjuntura, o Brasil conta com o Programa Saúde na Escola (PSE), cujo principal objetivo é contribuir com a formação integral de crianças e adolescentes em fase escolar, por meio de ações multiprofissionais de promoção da saúde, prevenção de doenças e atenção à saúde, com vistas no monitoramento e enfrentamento dos fatores de risco que comprometem o pleno desenvolvimento de crianças e jovens. Um dos seus pontos de maior interesse está no fomento à integração entre distintos profissionais dos setores educação e saúde nas fases de planejamento, condução e avaliação das ações².

O Decreto Presidencial n ${ }^{\circ}$ 6.286/2007 instituiu o PSE como uma política pública, desenvolvida a partir da articulação entre os Ministérios da Educação e da Saúde. Nesse sentido, cabe apresentar que o PSE caminha para além de uma estratégia de integração das políticas setoriais, visto que se propõe a ser um novo desenho da política de educação em saúde, por: a) tratar a saúde e a educação de forma integral, como parte de uma formação ampla para a cidadania e o usufruto pleno dos direitos humanos; b) permitir a progressiva ampliação das ações executadas pelos sistemas de saúde e educação com vistas à atenção integral à saúde de crianças, adolescentes e jovens e à educação em saúde e; c) promover a articulação de saberes, a participação de alunos, pais, comunidade escolar e sociedade em geral na construção e controle social da política ${ }^{3}$.

O PSE visa o enfrentamento das vulnerabilidades que comprometem o desenvolvimento de escolares da rede pública de ensino, propõe o atendimento a partir de um olhar multifacetado com a participação de profissionais das diversas áreas de conhecimento numa dinâmica multiprofissional e interdisciplinar ${ }^{4}$. Nesta perspectiva, é objetivo do presente trabalho descrever uma experiência de avaliação multiprofissional de saúde ocorrida em uma escola pública de ensino médio na cidade de Altamira-PA, que teve como principal fim identificar fatores de riscos e/ou doenças na população de interesse.

As escolas de médio e grande porte oferecem uma grande demanda para avaliações diagnósticas, especialmente de saúde, ação que exige também uma equipe com competência técnica. Dentre os profissionais que atuam na unidade escolar, apenas o profissional de educação física (PEF) possui tal qualificação, com reconhecimento profissional legal nas áreas de educação e saúde. Todavia, o pequeno número de PEF na equipe escolar é insuficiente para atender a demanda de escolares que precisam ser avaliados, considerando-se também outros fatores, como:o limitado tempo semanal oferecido ao componente (duas aulas semanais, que totalizam 100 minutos) e o elevado número de adolescentes das turmas (entre 35 e 40 discentes por sala). 
Com base nas demandas existentes, propôs-se a articulação intersetorial e a presença de equipe multiprofissional de saúde no âmbito escolar ${ }^{5-9}$. O grande desafio foi conciliar de forma concomitante as atividades curriculares obrigatórias com o desenvolvimento da ação de saúde.

\section{Descrição da experiência}

\section{Aproximação entre os profissionais e delimitação das ações}

Como primeiro passo, a proposta foi apresentada aos profissionais da escola pública, à coordenação da atenção básica (CAB) e equipe do PSE, no sentido de, além de abrir um canal de comunicação direta e pactuação entre os profissionais dos distintos setores, iniciar uma reflexão sobre os limites de ação ao longo da atividade.

Em um segundo momento, a apresentação da proposta foi estendida aos profissionais que atuam na unidade de saúde. Neste encontro, foi composta a equipe de ação, que contou com a presença da enfermeira, da técnica de enfermagem, duas agentes comunitárias de saúde (ACS), uma odontóloga com uma assistente, uma psicóloga, duas nutricionistas e uma assistente social. Na ocasião, também ocorreram as primeiras reflexões da equipe sobre o público alvo, os espaços disponíveis para as ações, as avaliações que seriam realizadas e a dinâmica do atendimento.

A PEF apresentou as características da rotina da escola, a meta de avaliação diagnóstica pretendida, assim como a projeção das ações que seriam realizadas no ambiente escolar, buscando uma analogia à reprodução da lógica do Sistema Único de Saúde (SUS), partindo da triagem até a especialidade. Na ocasião, as ações foram discutidas e pactuadas pela equipe de ação.

Na escola, os professores foram informados previamente sobre o período da ação e houve liberação dos alunos, em pequenos grupos, para fazê-la, no sentido de não haver prejuízos à dinâmica da escola. Nesse sentido, a ação e as aulas regulares aconteceram simultaneamente, estabelecendo-se que o tempo de cada adolescente na triagem não se excederia a uma hora, à exceção dos que posteriormente seriam encaminhados ao atendimento especializado.

Logo abaixo é apresentado fluxograma da organização do trabalho realizado (Figura 1).

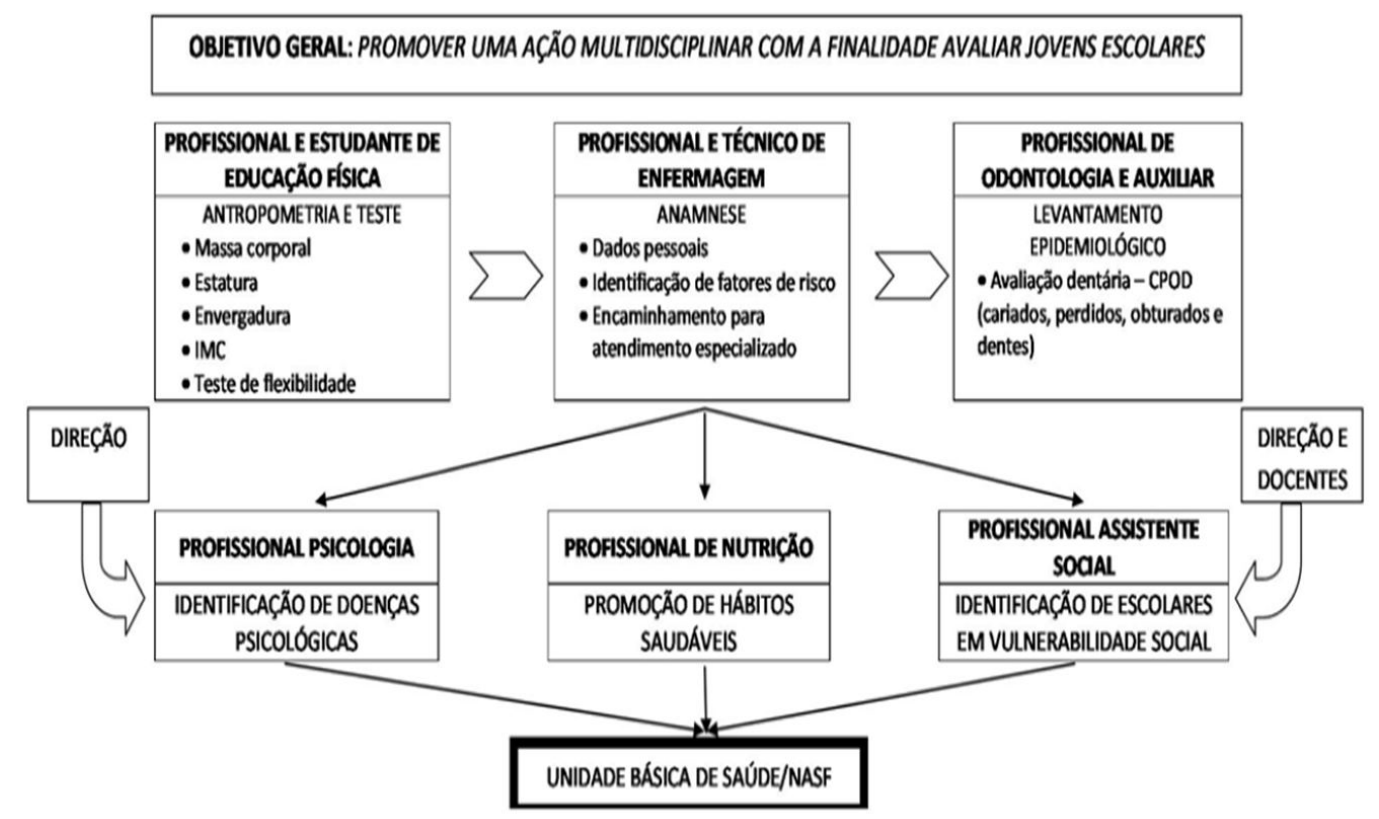

Figura 1. Organização do Trabalho Multiprofissional na Escola.

Fonte: Próprio autor. 
Organização e dinâmica das estações de passagem

Diante da necessidade de utilização dos espaços disponíveis na escola, estipulou-se que a triagem inicial, a avaliação física (ex. aferição de massa corporal e da estatura para determinação do índice de massa corporal, medida da envergadura e teste de flexibilidade), conduzida por discentes do curso de Educação Física da Universidade do Estado do Pará, sob a supervisão de um docente e anamnese (baseada em informações pessoais, de familiares e hábitos cotidianos, mais a aferição da pressão arterial, conduzida pela enfermeira auxiliada pelas ACS) seriam realizadas no mesmo espaço, em uma ampla sala de leitura.

Nesse mesmo espaço, houve o atendimento odontológico, objetivando o trabalho de educação preventiva para uma boa higiene bucal e diagnóstico das condições dentais por meio da ficha "CPO-D" (Dentes Cariados, Perdidos e Obturados), conforme faixa etária, pela odontóloga e sua auxiliar. Os casos identificados como graves foram encaminhados para tratamento especializado, orientados sobre os procedimentos necessários (documentação e acompanhamento de responsável) e agendados para tratamento junto à unidade básica de saúde próxima à localidade de moradia do escolar.

Para um melhor fluxo das ações e organização dos adolescentes, a dinâmica no espaço da sala de leitura foi organizada no formato de "estações de passagem" sequenciais, em ordem predisposta: avaliação antropométrica, teste de flexibilidade, anamnese e avaliação odontológica. Dessa forma, no momento da entrada dos adolescentes na sala de leitura, todos receberam um questionário que seria preenchido pela equipe e encaminhado à primeira "estação", para a aferição da medida de massa corporal, estatura e teste de flexibilidade.

Nesse momento se indicaria ou não a necessidade de encaminhamento do adolescente às outras especialidades (e.g. psicólogo, nutricionista e assistente social).

\section{Dinâmica do atendimento especializado}

Resguardando-se a privacidade dos escolares, salas individuais foram disponibilizadas para atendimentos da psicóloga, assistente social e nutricionista. Mesmo que a maior parte dos adolescentes tenha apresentado interesse nos atendimentos da nutricionista, observou-se também boa aceitação aos demais encaminhamentos.

No ato do atendimento com a psicóloga, o relato de vulnerabilidades sociais (ex. casos de abandono da família, perda traumática dos pais, dificuldade de interação social na escola) e/ou econômicas foram os principais indicadores para o encaminhamento à assistente social. Por outro lado, mesmo que não seja uma atribuição docente, as observações compartilhadas pelos professores da escola também foram importantes para a realização de encaminhamentos à assistente social, em vista da contínua convivência, ciência da realidade contextual e acompanhamento diário do desempenho de cada escolar.

Ao passo que a psicóloga identificava alguma situação de risco ou mesmo a ocorrência de transtornos/doenças psicológicas, esta era comunicada à coordenação pedagógica, que, em um segundo momento, informava a família. Em casos mais específicos (ex. tentativa de suicídio, depressão grave, quadros de anorexia ou bulimia), o atendimento foi estendido ao(s) familiar(es), recebendo encaminhamento para o Núcleo Ampliado de Saúde da Família (NASF) e o escolar para o Centro de Apoio Psicossocial Infantil (CAPSi).

Vale ressaltar que alguns dos encaminhamentos feitos à psicóloga foram a pedido da direção e coordenação pedagógica da escola, considerando-se os registros de ocorrências e a manifestação (ou suspeita) do uso de algum tipo de substância psicoativa (ex. cigarro, álcool, maconha) ou comportamento agressivo, violento na escola. Nesses casos, padrões permanentes de comportamentos de risco poderiam 
também ser indicativos para o encaminhamento à psicóloga. Todavia, em nenhum destes casos, se observou manifestação problemática de uso destas substâncias.

Para o atendimento nutricional foram realizadas "oficinas nutricionais", com grupos de até cinco escolares, que relatavam a sua rotina alimentar diária, o número de refeições diárias, suas preferências alimentares, se comiam frutas e legumes, quais eram os mais ingeridos, se comiam biscoitos recheados, sanduiches, bebiam refrigerantes, água, suco, etc. É importante pontuar que um dos principais pontos indicados foi que boa parte dos adolescentes não se alimentavam no momento prévio à entrada na escola, dependendo, exclusivamente da merenda escolar.

Mesmo em posse dos relatos individuais, as orientações nutricionais foram realizadas em grupo, baseando-se na preferência (ou disponibilidade) pelo consumo de alimentos saudáveis, como: frutas, legumes, hortaliças, sucos e, também, orientação sobre o uso de suplementos alimentares nos casos de adolescentes praticantes de exercício físico, que informaram seu consumo.

Nos atendimentos especializados também foram questionadas a capacidade visual dos adolescentes, com orientação para realização do teste de acuidade visual (SNELEN), dificuldade auditiva e condições especiais não caraterizadas pelo profissional. Dessa forma, evidenciou-se a importância de um médico (clínico geral) para a realização de diagnósticos mais precisos em determinados casos junto à equipe multiprofissional, assim como da presença de um terapeuta ocupacional para auxiliar nas ações junto aos adolescentes com transtornos neuropsicomotores, por exemplo.

\section{Resultados e impactos}

\section{Pontos potenciais e limitadores da experiência}

A baixa aceitação de alguns professores da escola foi um dos principais limitadores da proposta. Esse foi um ponto muito contraditório, pois a avaliação multiprofissional estava prevista entre os eventos que aconteceriam logo no início do ano letivo, de modo que houvesse tempo hábil para o acompanhamento e intervenções pedagógicas necessárias.

Parte desta baixa aceitação pode ser justificada pela preocupação em prejudicar o andamento das aulas, o que comprometeria o conteúdo a ser ministrado. A estratégia de chamar os adolescentes por grupos (entre 10 e 15 adolescentes por vez) foi adotada para tentar minimizar problemas na dinâmica das aulas regulares, de modo que nem todos se ausentariam ao mesmo tempo. Logo que finalizavam as avaliações, os adolescentes sem necessidade de atendimento especializado retornavam à sala de aula.

Houve dificuldade para associar o atendimento dos profissionais de saúde na escola com o da unidade básica de saúde (UBS), diante da elevada demanda no centro de saúde. $\mathrm{ACAB}$ apresentou uma escala de atendimento que variou conforme a disponibilidade e dia de trabalho do profissional, de modo que foi realizado um revezamento do atendimento entre a escola e a UBS. A articulação e as reuniões prévias foram fundamentais para fortalecer as ações, pois, também se fez necessário pensar nas aulas que não poderiam ser interrompidas, mantendo o fluxo contínuo da escola, onde se estabeleceu que as avaliações da turma não poderiam ocorrer e coincidir com as aulas do mesmo professor, por duas vezes consecutivas, para não prejudicar o conteúdo a ser ministrado na turma.

Outro fator relevante foi estruturar a agenda da UBS para o atendimento especializado da demanda da escola, pois foram avaliados 562 escolares (55\% do sexo feminino), com faixa etária entre 14 e 17 anos (média de 15,8 anos). Visto a inexistência de critérios de inclusão e exclusão, todos e todas as adolescentes foram avaliados, sendo seus pais/ responsáveis também avisados previamente sobre a ação. Destes, 
328 (58\% dos avaliados), foram encaminhados à UBS para atendimento especializado (52\% do sexo feminino).

No sentido operacional, coube à PEF coordenadora da ação: I) organizar previamente os materiais e o fluxo das ações, II) articulação, junto à direção escolar e equipe de saúde e III) acompanhamento dos atendimentos. A ação também contou com o auxílio dos representantes das turmas, que em muito ajudaram na divulgação da ação e organização dos seus colegas de sala, da sala de aula até o local de avaliação. Sua participação foi fundamental para o bom desenvolvimento das ações, muito por conta da proximidade e da liberdade de comunicação para com seus colegas.

Uma das limitações identificadas no processo foi a timidez do escolar mediante as avaliações coletivas, que ocorreram no mesmo espaço (sala de leitura). Para minimizar tal desconforto, foram fornecidas orientações aos escolares, informando-os previamente sobre o que seria avaliado e sobre o sigilo das informações coletadas. Foi permitida a formação de grupo conforme sua afinidade e relação de confiança. Avaliamos que um dos pontos altos da ação foi a realização de um momento de devolutiva e reflexão após as coletas diárias com os profissionais envolvidos, no sentido de reforçar a dinâmica do trabalho e buscar minimizar eventuais inadequações nas coletas futuras.

Os dados quantitativos coletados pela equipe foram inseridos no sistema eletrônico de informação do SUS (e-SUS). Pontos destacados em relação ao e-SUS é que ele não faz distinção por área, assim como não permite buscar dados de locais específicos para reanálise. Isso se configura como uma dificuldade para comparar resultados conforme o território de cobertura. Outro fator relevante, seria a inclusão do relatório discursivo (avaliação qualitativa) das ações desenvolvidas, como ocorreu o gerenciamento dos problemas e soluções encontradas, de modo a nortear a tomada de decisão e embasar futuras ações.

\section{Considerações finais}

Diante da importância do PSE, na promoção da saúde das crianças e adolescentes que vivem no país, buscou-se compartilhar, por meio do presente relato, a realização de uma ação intersetorial de interesse, que resultou em benefícios, nos diversos níveis: I) aos escolares, o acesso (e conhecimento) ao serviço de saúde, o reconhecimento da sua condição de saúde, assim como a possibilidade de encaminhamento para o tratamento dos agravos identificados; II) para a escola, como um todo, a obtenção de um diagnóstico de saúde dos escolares, de modo que estas informações possam se reverter em maior autonomia para intervir em situações que comprometam o rendimento escolar; III) aos profissionais da saúde, para além da maior inserção na escola, atendendo um público específico e de grande importância nas ações em saúde, a possibilidade do estreitamento intersetorial, de modo que futuras ações possam ser desenhadas nesta perspectiva e; IV) à $C A B$, que propiciou o acesso aos serviços, utilizando a ação como porta de entrada para o atendimento no SUS, fomentando o cuidado à saúde na população de adolescentes matriculados na escola em questão.

\section{Agradecimentos}

A Kátia Mirella da Silva Lopes e Loreane Mayara Santos Corrêa, pela colaboração para o desenvolvimento da ação.

\section{Referências}

1. Carvalho FFB. A saúde vai à escola: a promoção da saúde em práticas pedagógicas. Physis. 2015;25(4):1207-27. http:// dx.doi.org/10.1590/S0103-73312015000400009. 


\section{ighe}

2. Brasil. Ministério da Saúde. Secretaria de Atenção à Saúde. Departamento de Atenção Básica. Saúde na escola. Brasília: Ministério da Saúde; 2009. 96 p. (Série B. Textos Básicos de Saúde) (Cadernos de Atenção Básica; no. 24).

3. Brasil. Presidência da República. Decreto ${ }^{\circ}$ 6.286, de 5 de dezembro de 2007. Institui o Programa Saúde na Escola - PSE, e dá outras providências. Diário Oficial da União, Brasília, DF, 6 dez. 2007.

4. Brasil. Ministério da Saúde. Booklet of the School Health Program (SHP) manager [Internet]. Brasília: Ministério da Saúde; 2015 [citado em 22 Jan. 2019]. Disponível em: http://bvsms.saude.gov.br/bvs/publicacoes/caderno_gestor_pse.pdf.

5. Sales WB, Oliveira ASC, Pereira LEA, et al. A importância da equipe NASF/AB - enfrentamentos e multidisciplinariedade: uma revisão narrativa/crítica. Revista Eletrônica Acervo Saúde. 2020;48(supl 48):e3256-3256. http://dx.doi.org/10.25248/ reas.e3256b.2020.

6. Lotti AD, Nakamura E. Significados da prática profissional em Educação Física na área da saúde. Pensar a Prática. 2020;23:e54518. http://dx.doi.org/10.5216/rpp.v23.54518.

7. Silva CS, Bodstein RC. Referencial teórico sobre práticas intersetoriais em Promoção da Saúde na Escola. Cien Saude Colet. 2016;21(6):1777-88. http://dx.doi.org/10.1590/1413-81232015216.08522016. PMid:27281663.

8. Silva CS. Promoção da saúde na escola: modelos teóricos e desafios da intersetorialidade no Município do Rio de Janeiro [doutorado]. Rio de Janeiro (RJ): Escola Nacional de Saúde Pública; 2010. 198 p.

9. Donato LMTM, Moraes SHM, Nunes CB, Gerk MAS. A interação entre ESF e escola na saúde do adolescente. Cadernos ABEM. 2012;8(1):13-9.

\section{Contribuição dos autores}

Dinar Duarte Vasconcelos foi idealizadora e coordenadora geral da ação, trabalhou no planejamento e execução da ação, na concepção, revisão, análise, organização da ilustração e redação final do manuscrito. Paulo Henrique Guerra fez a orientação da escrita, contribuiu com a revisão crítica, análise e redação final do manuscrito. Jeniffer Fagundes contribuiu com a transcrição dos dados e pesquisa bibliográfica. Kharinni Uchôa Pereira contribuiu com a pesquisa bibliográfica e escrita do manuscrito Silvia Ribeiro Santos Araújo contribuiu com a escrita, revisão, tradução e organização da ilustração 